\title{
2. Sicherheit als sozialer Prozess: Zum theoretischen Analyserahmen der Studie
}

Bürgerwehren führen eine »Unsicherheit« als Grund für ihr Bestehen an und versprechen, mit ihren Patrouillen »Sicherheit« zu schaffen. Diese Selbstverortung macht Sicherheit zum zentralen Ausgangspunkt für ihre Untersuchung. Wer erklären möchte, wieso das Phänomen Bürgerwehren auftritt, kommt um die Sicherheit nicht herum. Deshalb wird für diese Arbeit ein theoretischer Rahmen aus der Sicherheitsforschung gewählt.

In den Security Studies der Politikwissenschaft waren lange Zeit Staaten die zentralen Akteure, das internationale Staatensystem der Analyserahmen, und Sicherheit wurde vor allem materialistisch definiert. Bis in die 1990er Jahre waren diese neorealistischen Ansätze in der europäischen und US-amerikanischen Wissenschaft der internationalen Beziehungen vorherrschend. Die Werte oder Identitäten der handelnden Individuen spielten keine Rolle für die Erklärung staatlichen Handelns. Erst mit dem Ende des Ost-West-Konflikts, als der Neorealismus an Erklärungskraft verlor, fanden auch liberale, institutionalistische und (moderat) konstruktivistische Ansätze Beachtung.

Diese Ansätze nahmen daraufhin zwar nicht-staatliche Akteure in ihre Theorien auf, ${ }^{1}$ doch behandelten sie diese getreu der neorealistischen Herangehensweise als uniforme rationale Akteure. ${ }^{2}$ In den USA fanden vor allem die Konstruktivismen nach Alexander Wendt (Anarchy is what States make of

1 Vgl. Katzenstein, P. J. (Hg.) (1996): The culture of national security: norms and identity in world politics. New York: Columbia Univ. Press. S. $524 \mathrm{f}$.

2 Vgl. Hinz, Anne (2007): Security and the Costs and Benefits of Manipulating Analytical Boundaries: Constructivist Debates Within European Critical Security Studies. In: Sicherheit und Frieden (S+F)/Security and Peace 25 (4). Nomos. S. 202. 
it), Peter Katzenstein (The Culture of National Security) sowie Emanuel Adler und Michael Barnett (Security Communities) Gehör. ${ }^{3}$

Diese sogenannte »ideas literature« erweitert die positivistische Herangehensweise um den Faktor »Idee«, um Entwicklungen ab Ende des Kalten Kriegs erklären zu können, die dieser rationalistische Ansatz nicht fassen konnte. Dabei hinterfragt dieser konventionelle Konstruktivismus jedoch nicht die bestehenden Konzepte, sondern ergänzt diese lediglich um die Kategorien »Identität« und »Werte«. Diese Ansätze untersuchen zwar Identitäten und Werte, bleiben dabei aber dem rationalistischen Ansatz verhaftet, ohne über die Bedeutung von Sprache in sozialen Beziehungen zu reflektieren. ${ }^{4}$ Erst die Kritik an der »ideas literature « trug den »linguistic turn « auch in die Security Studies ein, "der letzten Bastion des neorealistischen Dogmas ${ }^{5}$

Warum aber eignet sich ein konstruktivistischer Ansatz überhaupt für die Analyse von Bürgerwehren? Zum einen existieren viele potentielle Bedrohungen, die eine Bürgerwehr als Gefahr benennen könnte, um ihr Bestehen zu rechtfertigen. Jedes Jahr sterben beispielsweise viele Fußgänger*innen bei Autounfällen - auf die Idee, die Fußgänger*innen vor den Autofahrer*innen zu schützen, ist jedoch noch keine Bürgerwehr gekommen. Im Gegenteil, oft sind die Gefahren, die von der Bürgerwehr als Gründe für ihr Entstehen angeführt werden, gar nicht nachprüfbar. So gründete sich in Rostock beispielsweise eine Bürgerwehr, um die Frauen und Kinder der Stadt vor einem Exhi-

Vgl. Wendt, Alexander (1992): Anarchy is What States Make of It: The Social Construction of Power Politics. In: International Organization. 46. S. 391-425. Katzenstein, P. J. (Hg.) (1996): The culture of national security: norms and identity in world politics. New York: Columbia Univ. Press. Adler, E., \& Barnett, M. (Hg.) (1998): Security Communities (Cambridge Studies in International Relations). Cambridge: Cambridge University Press.

4 Vgl. Hinz, Anne (2007): Security and the Costs and Benefits of Manipulating Analytical Boundaries: Constructivist Debates Within European Critical Security Studies. In: Sicherheit und Frieden (S+F)/Security and Peace 25 (4). Nomos. S. 203.

5 Krause, Keith, zitiert nach: Hinz, Anne (2007): Security and the Costs and Benefits of Manipulating Analytical Boundaries: Constructivist Debates Within European Critical Security Studies. In: Sicherheit und Frieden (S+F)/Security and Peace 25 (4). Nomos. S. 202. 
bitionisten zu schützen, dessen Existenz nicht zu beweisen war und den kein Mitglied der Bürgerwehr je selbst zu Gesicht bekommen hatte. ${ }^{6}$

Welcher Sachverhalt also von einer Bürgerwehr als existentielle Gefahr angeführt wird, gegen die es wichtig ist, auf den Straßen zu patrouillieren, hängt nicht vom materiellen Gehalt der Bedrohungen ab, sondern davon, wie diese von den Mitgliedern der Bürgerwehr und von der örtlichen Bevölkerung wahrgenommen wird.

Angst herrscht vor dem, was im gesellschaftlichen Diskurs als Bedrohung benannt wird. Angst ist nicht unbedingt ein objektiv begründbares Gefühl. So lässt sich beispielsweise beobachten, dass Menschen nach den Anschlägen vom 11. September 2001 mehr Angst vor einer Flugreise hatten als vor einer Autofahrt, obwohl sich diese Einschätzung nicht mit den statistisch beschreibbaren Bedrohungslagen deckte. ${ }^{7}$ Diese gefühlte Bedrohungslage in Bezug auf terroristische Anschläge erklärt auch, warum im Namen der Terrorismusbekämpfung Maßnahmen gerechtfertigt werden können, für die es ohne diese Angst keine Mehrheit gäbe.

Für die Untersuchung von Bürgerwehren eignet sich jedoch kein Konstruktivismus nach US-amerikanischer Fasson, wie er von Katzenstein oder Wendt vertreten wird. Diese Ansätze untersuchen zwar Identitäten und Werte, für eine Untersuchung von Bürgerwehren ist jedoch zentral, über die Bedeutung von Sprache in sozialen Beziehungen zu reflektieren. Denn eine Bürgerwehr kann nur überleben, wenn sie ihr Bestehen rechtfertigt - und das geschieht über Sprache. Diese Ebene kann die »ideas literature« nicht erfassen. Zudem ist bei der Untersuchung von Bürgerwehren der Referenzrahmen nicht das internationale Staatensystem, ob anarchisch oder nicht. Hier geht es um die gesellschaftliche Ebene. Der Sicherheitsbegriff muss also weiter gefasst sein. Und er muss gesellschaftliche Akteure zulassen, die nicht auf der Ebene des internationalen Staatensystems handeln.

Dieser konsequentere Konstruktivismus ist nicht in US-amerikanischen Theorieschulen zu finden, sondern in Europa. Hier hat sich seit Ende der warnow. In Berliner Zeitung vom 4.11.2014. Online unter: www.berliner-zeitung.de/bu ergerwehr-in-rostock-jagdszenen-an-der-unterwarnow-137896 (Zugriff am 2.1.2021). gen des erweiterten Sicherheitsbegriffs. In: Zoche/Kaufmann/Haverkamp (Hg.) (2010): Zivile Sicherheit. Gesellschaftliche Dimensionen gegenwärtiger Sicherheitspolitiken. Bielefeld: transcript. S. 150. 
1980er Jahre eine eigenständige Theoriedebatte entwickelt, die den linguistic turn auch in die Security Studies einträgt. Es ist vielleicht nicht verwunderlich, dass diese neue Sicherheitstheorie in Europa entstand: Denn im geteilten Europa konnte man beobachten, dass die Dynamik des Kalten Krieges nicht nur auf die Interessen von zwei Supermächten zurückzuführen war. ${ }^{8}$

Im Folgenden werden daher die Ansätze der New European Security Theory diskutiert und auf eine Anwendung auf das Phänomen Bürgerwehren geprüft. Die New European Security Theory lehnt den bis dato in den internationalen Beziehungen vorherrschenden substantiellen Sicherheitsbegriff ab. ${ }^{9}$ In ihrer akteursorientierten, diskurstheoretischen Beobachtung von Sicherheitspolitiken setzt sie sich deutlicher als die Konstruktivismen amerikanischen Typs von neorealistischen Ansätzen ab. Materielle Macht und deren ideelle Konstitutionsbedingungen werden hier nicht als Gegensätze gesehen, sondern erst in ihrem Wechselwirkungsverhältnis analysierbar. ${ }^{10}$

Fundamental unterscheiden sich die Theorien der New European Security Theory von den neorealistischen IB-Theorien in puncto Selbstreflexion. Die Fragen nach den unvermeidlichen Auswirkungen von politikwissenschaftlichen Theorien und dem damit verbundenen unvermeidlichen politischen Engagement von Theoretiker*innen werden von der New European Security Theory offensiv thematisiert.

Die New European Security Theory kritisiert, dass herkömmliche Sicherheitstheorien in das westliche Narrativ von Modernität eingebettet sind und dieses unhinterfragt reproduzieren. Auch wenn die drei Schulen der New European Security Theory diese kulturelle Voreingenommenheit reflektieren, bleiben doch auch sie derselben Erzählung verhaftet und können daher nicht ohne Weiteres auf Phänomene außerhalb Europas und US-Amerikas angewendet werden. Für die Untersuchung von Bürgerwehren in Deutschland eignet sich die Theorie der Versicherheitlichung jedoch trotzdem, denn der Untersuchungsgegenstand (die Gesellschaft) und die Akteure sind in Deutschland verortet.

Vgl. Hinz, Anne (2007): Security and the Costs and Benefits of Manipulating Analytical Boundaries: Constructivist Debates Within European Critical Security Studies. In: Sicherheit und Frieden (S+F)/Security and Peace 25 (4). Baden-Baden: Nomos. S. 204.

9 Vgl. Baldwin, D. A. (1995): Security Studies and the End of the Cold War. In World Politics. Princeton etc.: Cambridge University Press, S. 134.

10 Vgl. Büger, C./Stritzel, H. (2005): New European Security Theory. Zur Emergenz eines neuen europäischen Forschungsprogramms. In: Zeitschrift für Internationale Beziehungen. Baden-Baden: Nomos. S. $437 f f$. 
Im Folgenden werden also zunächst die drei Schulen der New European Security Theory vorgestellt und kritisch gegeneinander abgewogen. Daraufhin wird die Theorie der Versicherheitlichung für die Analyse von Bürgerwehren aufgeschlüsselt.

\subsection{Theorien der Versicherheitlichung: Die "New European Security Theory"}

Die New European Security Theory kristallisiert sich in drei Zentren der europäischen Politikwissenschaft heraus: Kopenhagen, Aberystwyth (Wales) und Paris, jedes mit unterschiedlichen Annahmen und Schwerpunkten. Die Einteilung der drei theoretischen Ansätze in Schulen geschah jedoch mehr durch Fremdzuschreibungen, als dass sie von den beteiligten Wissenschaftlern initiiert worden wäre. Es lässt sich jedoch nicht immer eine klare theoretische Linie zwischen den Vertretern der Schulen ziehen. ${ }^{11}$

Die Kopenhagener Schule um Barry Buzan und Ole Wæver gründet ihre Theorie der Versicherheitlichung auf die Sprechakttheorie von Austin und begreift Sicherheit als illokutionären Sprechakt, während die Waliser Schule, ${ }^{12}$ auch als Critical Security Studies bezeichnet, um Ken Booth, Richard Wyn Jones und Michael Williams einen normativen Ansatz vertritt, der sich an der Frankfurter Schule orientiert.

Die Pariser Schule schließlich baut auf Bourdieu und Foucault auf und wird vor allem von Didier Bigo repräsentiert. Dreh- und Angelpunkt seiner Sicherheitstheorie sind die security professionals, Akteure, die innerhalb bürokratischer Strukturen agieren. Damit ist die Pariser Schule institutionalistischer und soziologischer als die Kopenhagener und die Waliser Schule.

Diese Theoriedebatte und damit das Entstehen einer europäischen Sicherheitstheorie begann 1983 mit Barry Buzans Publikation People, States, and Fear. ${ }^{13}$ Die Kopenhagener Schule machte somit den Anfang und soll auch

11 Vgl. C.A.S.E. Collective (2006): Critical Approaches to Security in Europe: A Networked Manifesto. In: Security Dialogue vol 37, no. 4, December 2006. S. 450.

12 Weil die beiden anderen Schulen nach Städten benannt wurden, müsste bei der Waliser Schule korrekterweise eigentlich von der Aberystwythschen Schule gesprochen werden. Wohl, weil die Stadt in Wales nicht bekannt genug ist, hat sich jedoch die Bezeichnung Waliser Schule durchgesetzt. 
hier als erstes diskutiert werden. Danach werden die Waliser und die Pariser Schule jeweils in Bezug auf ihre Abgrenzungen und Gemeinsamkeiten zur Kopenhagener Schule diskutiert. Dabei zeige ich, dass die Kopenhagener Theorie der Versicherheitlichung für die Analyse von Bürgerwehren am besten geeignet ist.

\subsubsection{Securitization as performative action: Der konstruktivistische Ansatz der Kopenhagener Schule}

Die Kopenhagener Schule entstand aus einer Gruppe von Wissenschaftlern am Conflict and Peace Research Institute der Universität Kopenhagen. Als Hauptvertreter gelten Buzan und Wæver, die zwischen 1987 und 1998 fünf Hauptwerke einer »Neuen Theorie der Sicherheit« veröffentlichten. Die Debatte begann mit »Security. A New Framework of Analysis«. Das New im Titel weist bereits auf die Abgrenzung zu den herkömmlichen Security Studies hin.

Für die Kopenhagener Schule ist Sicherheit kein objektiver Zustand, sondern das Ergebnis eines sozialen Prozesses. Die Theorie der Versicherheitlichung untersucht den Prozess, mit dem eine Begebenheit zu einem Sicherheitsthema erhoben wird. Die Konstruktion von Sicherheit und Unsicherheit in den Blick zu nehmen, bedeutet, die politische Sprache in ihrer realitätskonstituierenden Wirkmächtigkeit zu begreifen. Ein vorherrschendes Narrativ, wie das vom »Kampf gegen den Terrorismus«, dient politischen Akteuren, innenpolitische Sicherheitsmaßnahmen zu rechtfertigen. ${ }^{14}$

Es gilt also $\mathrm{zu}$ untersuchen, wie eine konstruierte Unsicherheit dazu dient, strittige Gesetze zu rechtfertigen, etwa, wie im Zuge der Terrorismusbekämpfung in das Grundrecht auf informationelle Selbstbestimmung eingegriffen wird. Hier wird »der Terrorismus« als Entität konstruiert, die genauso bekämpft werden kann wie ein Staat. ${ }^{15}$ Analog beschreibt Holger Stritzel das Narrativ eines »Kampfes gegen die >organisierte Kriminalität««. ${ }^{16}$

14 Vgl. Schulze, Matthias (2012): Die Sprache der (Un-)Sicherheit. Die Konstruktion von Bedrohung im sicherheitspolitischen Diskurs der Bundesrepublik Deutschland. Band 51, Marburg: Tectum Verlag. $133 \mathrm{ff}$.

15 Vgl. Schulze, Matthias (2012): Die Sprache der (Un-)Sicherheit. Die Konstruktion von Bedrohung im sicherheitspolitischen Diskurs der Bundesrepublik Deutschland. Band 51, Marburg: Tectum Verlag. S. $11 \mathrm{f}$.

16 Vgl. hierzu: Stritzel, Holger (2011): Security as translation: threats, discourse, and the politics of localization. In: Review of International Studies, Vol. 37. S. 2491-2517. 
Mit der Beschreibung von Sicherheitsproblemen als soziale Konstruktionen rückt die Frage in den Mittelpunkt, auf welche Weise Themen zu Sicherheitsthemen werden. Das geschieht nach Wæver und Buzan durch einen Prozess der Versicherheitlichung. Dieser Prozess hat eine sprechakttheoretische Fundierung, er baut auf einem securitizing speech act auf, einem Sprechakt der Versicherheitlichung.

Die Theorie beruft sich dabei auf die Sprechakttheorie von John L. Austin und geht davon aus, dass Sprache nicht nur dazu dient, etwas zu beschreiben, sondern selbst eine Form des Handelns darstellt: "By saying the words, something is done. ${ }^{17}$ Die Bezeichnung eines Themas als Sicherheitsproblem stellt somit einen illokutionären Akt dar. Es ist eine Handlung, denn es positioniert das Thema in einer Art und Weise, die den Umgang mit dem Thema bestimmt. Durch die Ernennung zum Sicherheitsproblem kann ein Akteur besondere Maßnahmen zur Lösung des Problems einfordern:

»In naming a certain development a security-problem the sstate can claim a special right, one that will in the final instance, always be defined by the state and its elites. ${ }^{18}$

Hier zeigt sich die enorme Erweiterung des Sicherheitsbegriffs, die die Kopenhagener Schule vornimmt. Prinzipiell kann jedes Problem zum Sicherheitsproblem werden. Austin beschreibt jedoch Bedingungen, die gegeben sein müssen, damit ein Sprechakt erfolgreich ist. Und ebenso formulieren auch Buzan, Wrver und de Wilde Bedingungen, damit die Versicherheitlichung erfolgreich ist. Akteure haben je nach ihrer sozialen Position unterschiedliche Möglichkeiten, sozial effektive Behauptungen zur Sicherheit zu machen. Dadurch wird securitization bis zu einem gewissen Grade ein vorhersehbarer Akt.

Der Sprechakt der Versicherheitlichung wird getätigt, um einer Bedrohung zu begegnen. Was aber als Bedrohung angesehen wird, ist Ergebnis eines sozialen Aushandlungsprozesses.

»Issues become ssecuritized،, treated as security issues, through these speech-acts, which do not simply describe an existing security situation, but 
bring it into being as a security situation by successfully representing it as such. ${ }^{19}$

Der Versicherheitlichungsprozess erfolgt in zwei Stufen, der stage of identification und der stage of mobilization. ${ }^{20}$ Die erste Stufe ist linguistischer Natur. Hier nimmt der securitizing actor eine Bedrohung wahr und artikuliert diese. Diese Bedrohung muss als ein existential threat präsentiert werden, eine Gefahr, die so bedeutend ist, dass alles andere irrelevant ist, solange sie nicht gebannt wird. Nach dem Motto: Wenn wir hier nicht zu außergewöhnlichen Maßnahmen greifen, wo dann ${ }^{21}$

Auf der linguistischen Stufe des Prozesses stehen also Akteur, Bedrohung und Referenzobjekt in Beziehung zueinander. Ein Akteur (securitizing actor) benennt eine Gefahr (existential threat) für etwas (reference-object). Zwischen Referenzobjekt und Bedrohung besteht ein Konstitutionsverhältnis, das erst durch den/die Sprecher*in (securitizing actor) hergestellt wird. ${ }^{22}$ Die zweite Stufe des Versicherheitlichungsprozesses, die stage of mobilization, folgt, wenn der Sprechakt erfolgreich war. Dann bestehen Dringlichkeiten, der Bedrohung zu begegnen. Diese bilden die Basis für die extraordinary measures. Das ist die zweite Stufe des Versicherheitlichungsprozesses: die Akzeptanz von Sicherheitsmaßnahmen und deren Implementierung.

$\mathrm{Ob}$ eine Versicherheitlichung erfolgreich ist, entscheidet nicht der/die Sprecher"in (securitizing actor), sondern das Publikum (audience). Nur wenn der/die Sprecher*in das Thema hegemonial im Diskurs platziert, kann eine Versicherheitlichung erfolgreich sein. ${ }^{23}$ Es hängt also zentral von der Sprecher*innenposition $a b$, ob das Publikum den Sprechakt des Akteurs anerkennt. Dabei müssen auf einer internen Ebene die Aussagen des Akteurs linguistisch-grammatikalisch den Regeln des Sprechaktes folgen, und auf

19 Williams, Michael C. (2003): Words, Images, Enemies: Securitization and International Politics. In: International Studies Quarterly, Vol. 47. S. 513.

20 Vgl. Williams, Michael C. (2003): Words, Images, Enemies: Securitization and International Politics. In: International Studies Quarterly, Vol. 47. S. $20 \mathrm{ff}$.

21 Vgl. Buzan/Wæver/de Wilde (1998): Security. A New Framework for Analysis. London/Colorado: Rienner. S. 106.

22 Vgl. Schulze, Matthias (2012): Die Sprache der (Un-)Sicherheit. Die Konstruktion von Bedrohung im sicherheitspolitischen Diskurs der Bundesrepublik Deutschland. Band 51, Marburg: Tectum Verlag. S. 34.

23 Vgl. Bonacker, T./Bernhardt, ]. (2014): Von der security community zur securitized community. In: Siedschlag, A. (2014): Methoden der sicherheitspolitischen Analyse. Eine Einführung, Wiesbaden: VS Verlag. S. 245. 
Abbildung 2: Der Akt der Versicherheitlichung

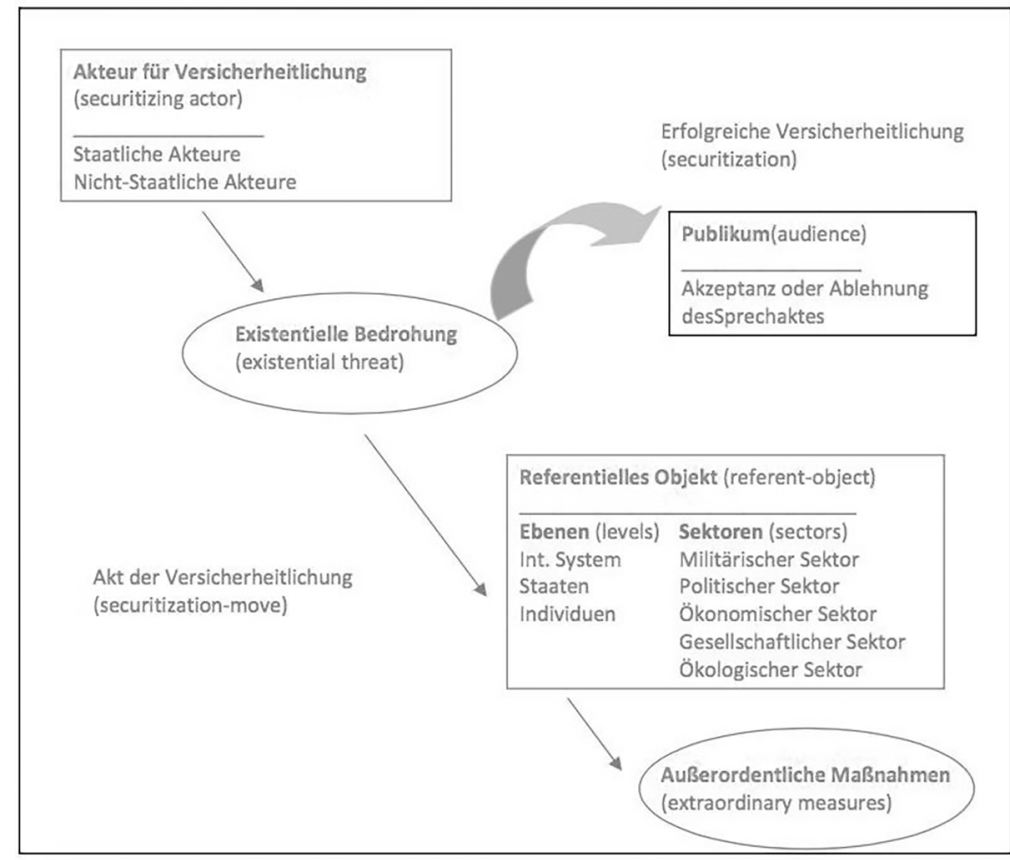

Quelle: Fischer/Klüfers/Masala/Wagner (2014): (Un-)Sicherheitswahrnehmungen und Sicherheitsmaßnahmen im internationalen Vergleich. In: Schiller/Gerhold/Steiger/Jäckel: Forschungsforum Öffentliche Sicherheit. Schriftenreihe Sicherheit Nr. 14, März 2014. S. $22 f$.

den sozialen Kontext bezogen, also extern, muss der/die Sprecher*in eine Position innehaben, von der aus sie gehört wird. Ihre Aussagen über die existentielle Bedrohung müssen rhetorisch, diskursiv, kulturell, institutionell zum Publikum passen. ${ }^{24}$

Es ist umso wahrscheinlicher, dass das relevante Publikum die Platzierung eines Themas als Sicherheitsbedrohung akzeptiert, je höher der soziale Status der Sprecher*innen innerhalb des Zielpublikums ist, das vom Sprech-

24 Vgl. Williams, Michael C. (2003): Words, Images, Enemies. Securitization and International Politics. In: International Studies Quarterly, Vol. 47. S. 514. 
akt überzeugt werden soll. ${ }^{25}$ Die Äußerungen des securitizing actors müssen auf Resonanz stoßen, sie müssen gehört werden. Findet der Sprechakt keine Resonanz beim Zielpublikum, bleibt der Versicherheitlichungsprozess unabgeschlossen.

Der Sicherheitsbegriff der Kopenhagener Schule ist prozessual, relational und konstruktivistisch. Sicherheit ist kein Zustand, sondern wird in einem sozialen Prozess hergestellt. Relational, weil er immer eine Verbindung zwischen dem securitizing actor und dem referent object erzeugt. ${ }^{26}$ Der Sicherheitsbegriff wird konstituiert durch den Sprechakt. Auf den ersten Blick erscheint er dadurch geradezu fahrlässig weit. So kann prinzipiell jedes Thema zum Sicherheitsthema erhoben werden, wenn es nur im Sprechakt als ein solches benannt wird.

Doch ist der Sicherheitsbegriff durch den Sprechakt auch gleichzeitig begrenzt. Denn der Sprechakt kann auch scheitern. Nicht jede Äußerung über Sicherheit führt zu einer Versicherheitlichung. Im Gegenteil: Die allermeisten Äußerungen verhallen im Alltagsrauschen des Diskurses. Genauso wie Aus$\operatorname{tin}^{27}$ und Searle ${ }^{28}$ Bedingungen für den Erfolg des Sprechakts formulieren, so geben auch Buzan und Wæver Kriterien an, damit eine Versicherheitlichung erfolgreich ist. Buzan und Weaver unterscheiden hierbei zwischen inneren und äußeren Bedingungen. Die inneren Bedingungen beziehen sich auf die Form und den Inhalt der Aussage. Sie muss dem Publikum, das es zu überzeugen gilt, und dem Sachverhalt, den es zu versicherheitlichen gilt, angemessen sein. Die äußeren Bedingungen beziehen sich auf den/die Sprecher* in und seine/ihre soziale Position innerhalb der Gesellschaft. Diese muss derart gestaltet sein, dass sie dem/der Sprecher*in Glaubwürdigkeit und Autorität bezüglich dem zu versicherheitlichenden Sachverhalt verleiht. ${ }^{29}$

25 Vgl. Schulze, Matthias (2012): Die Sprache der (Un-)Sicherheit. Die Konstruktion von Bedrohung im sicherheitspolitischen Diskurs der Bundesrepublik Deutschland. Band 51, Marburg: Tectum Verlag. S. 32.

26 Vgl. Bonacker, T./Bernhardt, ]. (2014): Von der security community zur securitized community. In: Siedschlag, A. (2014): Methoden der sicherheitspolitischen Analyse. Eine Einführung. Wiesbaden: VS Verlag. S. 245.

27 Vgl. Austin, John Langshaw (1972): Zur Theorie der Sprechakte. Stuttgart. S. 36-43.

28 Vgl. Searle, John R. (1971): Sprechakte. Ein sprachphilosophischer Essay. Frankfurt a.M.: Suhrkamp. S. 84.

29 Vgl. Klüfers, Philipp (2014): Internationale Sicherheit: Neuere Ansätze 3. In: Enskat, S./Masala, C. (2014): Internationale Sicherheit, Wiesbaden: Springer Fachmedien. S. 194f. 
Die Anforderungen an den/die Sprecher* in und an den Sprechakt begrenzen den Sicherheitsbegriff - es besteht dabei aber auch die Gefahr, dass durch die rhetorischen, diskursiven, kulturellen und institutionellen Anforderungen ein so enges Raster gezogen wird, dass die möglichen Aussagen zu einem deterministischen Verständnis dessen, was zu einem versicherheitlichenden Thema werden kann, führt.

Die Sprecher*innen besitzen in der Theorie der Versicherheitlichung dennoch agency, denn die Anforderungen verknappen vor allem die Sprecher*innen, nicht jedoch die Themen. Diese müssen zwar vorher politisiert worden sein, aber darauf können die Sprecher*innen Einfluss nehmen. Außerdem können sie wählen, ob sie ein politisiertes Thema zum Sicherheitsthema erheben wollen oder nicht. Denn Sicherheit ist im Konzept der Versicherheitlichung kein positiver Wert an sich, den es zu erlangen gilt. Vielmehr ist Versicherheitlichung ein Prozess, der aus einer bestimmten Motivationslage heraus eingeleitet wird, um sicherheitspolitische Maßnahmen durchzusetzen und der daher nur mit großer Vorsicht genutzt werden sollte.

Der Sprechakt ist in der Theorie der Versicherheitlichung ein abgeschlossener Akt, sein Anfang und sein Ende sowie die in ihm wirkenden Akteure sind klar definiert. Diese Sichtweise kritisiert beispielsweise Holger Stritzel, der dem kopenhagenschen Sprechakt in Anlehnung an Judith Butler eine prozesshafte kulturelle Praxis entgegensetzt. ${ }^{30}$ Die Versicherheitlichung des Themas ১organisierte Kriminalität` sei beispielsweise kein klar begrenzter Akt mit eindeutig zu benennenden Sprecher*innen und Publika, sondern »a complex, transnational, intertextual and gradually intensifying process that involved multiple actors, audiences and performative effects in various discoursive locals worldwide. ${ }^{31}$ Buzan und Wævers Konzeptionalisierung mag im Vergleich holzschnittartig wirken, jedoch ermöglicht sie eine Operationalisierung der Theorie, die mit einer prozessualen Sichtweise des Sprechaktes nur schwer möglich ist.

Auf der Kritik am Sprechakt aufbauend formuliert Stritzel eine generelle Kritik an der Theorie der Versicherheitlichung. Ihm zufolge bleibt ihre Herangehensweise im Kern den traditionellen/neorealistischen Annahmen verhaf-

30 Vgl. Stritzel, Holger (2007): Towards a Theory of Securitization. Copenhagen and Beyond. In: European Journal of International Relations, Vol. 13(3). S. $375 f$.

31 Stritzel, Holger (2011): Security, the translation. In: Security Dialogue 42: 4/5. S. 351. 
tet. Sein Vorwurf: Weaver et al. ignorierten in ihrem Konzept von Sicherheit dessen historische Genese. ${ }^{32}$

»It is ahistorical backwards because it overlooks the genealogy and historical contingency of the concept security. (...) Wæver's conceptualization is also ahistorical forwards because he >closes the meaning of security by fixing it as a spolitics of the exception<. In this sense the conceptualization is thus not only ahistorical but arguably also non-critical/anti-progressive, because it does not allow the meaning of security to progress beyond traditionalism/realism. «3

Diese Kritik wurde in ähnlicher Weise von einer "Second Generation [of] Securitization Theorists « geäußert. ${ }^{34}$ Im Zentrum dieser Weiterentwicklung der Theorie der Versicherheitlichung steht der Sprechakt, der prozesshafter und konsequenter konstruktivistisch konzipiert wird. Juha Vuori beispielsweise gründet ihr Verständnis des Sprechakts stärker auf Searles als auf Austins Ansatz und modifiziert die Theorie der Versicherheitlichung für ihre Anwendung auf nicht-demokratische Staaten. ${ }^{35}$ Thierry Balzacq formuliert eine fundierte Kritik am Akzeptanzbegriff der Theorie der Versicherheitlichung. ${ }^{36} \mathrm{Er}$ erweitert das in Teilen unterkomplexe Konzept der Akzeptanz des Sprechaktes durch die audience, indem er das Konzept der audience aufschlüsselt in:

»(i) [the] audience's frame of reference; (ii) its readiness to be convinced, which depends on whether it perceives the securitizing actor as knowing the issue and as trustworthy; and (iii) its ability to grant or deny a formal mandate to public officials. $\ll^{37}$

32 Vgl. ebd., S. 347.

33 Ebd., S. 347.

34 Vgl. ebd., S. 348.

35 Vgl. Vuori, Juha (2008): Illocutionary logic and strands of securitization: Applying the theory of securitization to the study of non-democratic political orders. European Journal of International Relations 14(1). S. 65-99.

Und: Vuori, Juha (2010): How to do security with words. A grammar of securitization in the People's Republic of China. Unpublished doctoral dissertation, Department of Political Science, University of Turku.

36 Vgl. Balzacq, Thierry (2005): The three faces of securitization: Political agency, audience and context. European Journal of International Relations 11(2). S. 171-201.

37 Ebd.. S. 192. 
Zudem fügt er mit der Kategorie des »Kontextes« den Erfolgschancen des Sprechaktes einen weiteren Einflussfaktor hinzu:

»Relevant aspects of the Zeitgeist that influence the listener, and the impact of the immediate situation on the way the securitizing author's sentences are interpreted by the listener. ${ }^{38}$

In eine ähnliche Richtung wie die Kritik von Stritzel, Balzacq und Vuori geht die Kritik an einer zu starren Konzeption der Sektoren. Eriksson beispielsweise kritisiert die Erweiterung des Sicherheitsbegriffs von der staatszentrierten, auf militärische Sicherheit fokussierten Herangehensweise der konventionellen Sicherheitstheorien hin zu einem Ansatz, der die Sektoren Ökonomie, Gesellschaft, Politik, Umwelt und Militär umfasst. In dieser Erweiterung liege, so Erikssons Vorwurf, eine gefährliche Ausweitung von Bedrohungen auf neue, vorher von Sicherheit und Unsicherheit unberührt gebliebene Bereiche.

»They (the Copenhagen School) might be seen as acting more as politicians than as analysts, objectifying security, and spreading the negative connotations of threat and enemies to new issue areas. ${ }^{39}$

Wæver reagierte, indem er darlegte, dass es nicht von der Definition der Sektoren abhänge, wieviele Versicherheitlichungsprozesse sich in den einzelnen Sektoren fänden, sondern von den handelnden Individuen.

»The set-up with five sectors is an analytical net to trawl through existing security discourses to register what is going on. ${ }^{40}$

Wæver erklärt geradezu unermüdlich, dass der Theorie der Versicherheitlichung eine Sicherheitsskepsis innewohne. Sicherheit habe keinen Wert an sich, und Versicherheitlichung sollte vermieden werden. Auch die Abkehr von der Staatenzentrierung in der Sicherheitstheorie und die daraus für die Kopenhagener Schule resultierenden fünf Sektoren führten nicht dazu, dass Versicherheitlichung wahrscheinlicher werde:

\footnotetext{
38 Ebd., S. 192.

39 Eriksson, Johan (1999): Observers or advocates? On the political role of security analysts. In: Cooperation and Conflict, 34 (3). S. 316.

40 Wæver, Ole (1999): Securitizing sectors? Reply to Eriksson. In: Cooperation and Conflict, 34 (3). S. 335.
} 
»We try conceptionally and definitionally to be open, to create a formal concept, and to let the world be state-centric or not, widening or not. Therefore, there is no contradiction between a sectoral approach and a wish to avoid securitizing. ${ }^{41}$

Die wahrscheinlich größte Kontroverse gab es um die Konzeption des Sektors society. Hier kritisierte vor allem Bill McSweeney, dass Buzan und Wæever Identität als das zentrale referent object von Versicherheitlichungen im Sektor society setzen würden. Diese Annahme sei politisch problematisch, weil die Theorie so Gefahr laufe, rechtes Denken zu legitimieren:

»If >security< is uncritically tied to a concern with >identity< and as a result reduced entirely to subjective judgment, then any xenophobic or racist vision of what is to be secured and from what (for instance, that of Le Pen and the National Front in France) seems both politically plausible and beyond what could be objected to. $\ll^{42}$

Darüber hinaus kritisiert McSweeney, die Kategorien Identität und Gesellschaft seien zu essentialistisch gedacht.

»Both >society< and sidentity< are here projected as objective realities, out there to be discovered and analyzed. $\ll^{43}$

In anderen Worten: Die Kopenhagener Schule sei nicht konstruktivistisch genug. ${ }^{44}$ Buzan and Wæver ließen mit einer Antwort auf McSweeneys Kritik nicht lange auf sich warten und wiesen die Vorwürfe entschieden zurück. McSweeneys Kritik resultiere aus einem mangelnden Verständnis der Theorie der Versicherheitlichung. Denn Identität sei bei Buzan und Wæver sehr wohl konstruktivistisch gedacht: ${ }^{45}$

41 Ebd., S. 335

42 Williams, Michael C. (1998): Modernity, Identity and Security: A Comment on the >Copenhagen Controversy<. In: Review of International Studies 24 (3). Cambridge University Press. S. $436 f$.

43 McSweeney, Bill (1996): Review: Identity and Security: Buzan and the Copenhagen School. In: Review of International Studies, Vol. 22, No. 1 (Jan. 1996). S. 83.

44 Vgl. Williams, Michael C. (1998): Modernity, Identity and Security: A Comment on the ,Copenhagen Controversy<. In: Review of International Studies 24 (3). Cambridge University Press. S. 436.

45 Vgl. Buzan/Wæver (1997): Slippery? contradictory? sociologically untenable? The Copenhagen school replies. In: Review of International Studies, Vol. 23. S. 243. 
»In our securitization perspective, identity is not a svalue< (i.e. the individual's), it is an intersubjectively constituted social factor. ${ }^{46}$

Dass Identität als Wert gesehen wird, der durch einen Sachverhalt (beispielsweise Migration) als bedroht erscheint, sei eine empirische Beobachtung, die mithilfe der Theorie der Versicherheitlichung analysierbar und kritisierbar werde. Versicherheitlichung ist bei Buzan und Wæver keine erstrebenswerte Entwicklung, im Gegenteil: Sie verstehen Versicherheitlichung als tendenziell konflikt-verschärfend. ${ }^{47}$

Die McSweeney-Debatte erfuhr in den 2000er Jahren eine Neuauflage. Francesco Ragazzi und Xavier Guillaume formulierten die Kritik am statischen Identitätsbegriff der Kopenhagener Schule neu. Ragazzi setzte diesem ein Konzept einer imagined community entgegen, welches Identität dynamisch und kontingent als Ergebnis eines fortwährenden sozialen Prozesses beschreibt. ${ }^{48}$ Während sich Ragazzi in seinem Gegenentwurf auf transnationale Identitätsbildungsprozesse in Diasporagemeinschaften konzentriert, erweitert Guillaume die Debatte mit seinem Konzept des symbolic citizenship. ${ }^{49}$

Buzan und Wæver entgegneten, wie auch schon in der McSweeneyDebatte, dass nicht die Theorie der Versicherheitlichung dieses statische Identitätskonzept $\mathrm{zu}$ verantworten habe, sondern Versicherheitlichungsprozesse dazu neigten, sich auf ein Identitätskonstrukt zu berufen, das holzschnittartig und anfällig für Ausgrenzungen und Nationalismen ist. Die starre Betrachtung von Identität als Entität wird also erst durch die Kopenhagener Schule sichtbar gemacht und somit kritisierbar. ${ }^{50}$

46 Ebd., S. 245.

47 Vgl. Bonacker, T./Bernhardt, ]. (2014): Von der security community zur securitized community. In: Siedschlag, A., (2014): Methoden der sicherheitspolitischen Analyse. Eine Einführung, Wiesbaden: VS Verlag. S. 246.

48 Vgl. Büger, C./Stritzel, H. (2005): New European Security Theory. Zur Emergenz eines neuen europäischen Forschungsprogramms. In: Zeitschrift für Internationale Beziehungen. Baden-Baden: Nomos. S. 441.

49 Vgl. Guillaume, Xavier/Huysmans, Jef (Hg) (2013): Citizenship and Security: The Constitution of Political Being. PRIO New Security Studies. Abingdon: Routledge.

50 Vgl. Büger, C./Stritzel, H. (2005): New European Security Theory. Zur Emergenz eines neuen europäischen Forschungsprogramms. In: Zeitschrift für Internationale Beziehungen. Baden-Baden: Nomos. S. 441. 


\subsubsection{Security as Emancipation: Der normative Ansatz der Waliser Schule}

Wie auch die Kopenhagener Schule grenzt sich die Waliser Schule radikal vom Neorealismus ab. Die vor allem auf die Arbeiten von Ken Booth und Richard Wyn Jones fußende Theorieschule kritisiert, dass der Neorealismus in seiner Staatenfixierung der Empirie nicht (mehr) gerecht werde. Denn in vielen Fällen sei es nicht ein anderer Staat, der die größte Gefahr für die Sicherheit einer Bevölkerung darstelle, sondern der eigene Staat, die Regierung oder gesellschaftliche Gruppen, die Gewalt ausübten. ${ }^{51}$

Auch Critical Security Studies genannt, rückt die Waliser Schule in der Tradition der Frankfurter Schule Emanzipation ins Zentrum ihrer normativen Theorie. Die Sicherheits-Macht-Normalität der herkömmlichen Sicherheitstheorien wird durch eine Sicherheit-Emanzipations-Normativität ersetzt. $^{52}$

Die Waliser Schule weist in Frankfurter Tradition auf die politische Rolle politikwissenschaftlicher Theorien hin: "If we insist upon old images, the future will naturally tend to replicate the past. $\ll^{53}$ Davon ausgehend, dass politikwissenschaftliche Theorie immer auf die Wirklichkeit einwirkt, folgern die Critical Security Studies, dass dieses Einwirken aktiv gestaltet werden sollte. Die Waliser Schule ist ein politisches Projekt. Ihre Vertreter wollen politisch auf die Welt einwirken, "to help create the political conditions for a more secure future. ${ }^{54}$ Zudem solle die Emanzipation auch innerhalb der akademischen Welt wirken:

»The aim is thus to free >mainstream< colleagues from their false consciousness of seeing security as belonging to the state and the military. $\varkappa^{55}$

51 Vgl. Wyn Jones, Richard (1995): >Message in a bottles? Theory and praxis in critical security studies. Contemporary Security Policy 16(3). S. 310.

52 Vgl. C.A.S.E. Collective (2006): Critical Approaches to Security in Europe: A Networked Manifesto. In: Security Dialogue vol 37, no. 4, December 2006. S. 456.

53 Booth, Ken (1991): Security and emancipation. In: Review of International Studies. London u.a: Cambridge University Press. S. 315.

54 Booth, Ken (2007): Theory of world security. Cambridge u.a.: Cambridge Univ. Press. S. 37.

55 Floyd, Rita (2007): Towards a consequentialist evaluation of security: bringing together the Copenhagen and the Welsh School of security studies. In: Review of International Studies (2007), Vol. 33, No 2. S. 333. 
Wie im Falle der Kopenhagener Schule stehen also auch die Vertreter der Waliser Schule fest auf konstruktivistischem Boden. Die Waliser Schule geht dabei sogar noch über die Kopenhagener Schule hinaus, indem sie der Annahme folgt: Wenn alles Wissen sozial produziert wird, so ist auch alles Wissen über Sicherheit kein objektives, sondern ein Abblild politischer Machtstrukturen. ${ }^{56}$ So sind nicht nur die Bedrohungen konstruiert, sondern auch die objects of security. Auch die Gesellschaft oder die Umwelt werden als sozial konstruiert angesehen. ${ }^{57}$ Die Kopenhagener Schule hingegen hat hier einen relativ objektivistischen Ansatz und geht von zumindest für den Moment der Versicherheitlichung fixen objects of security aus. Insbesondere für die objektivistische Herangehensweise an Gesellschaft und Identität erntete die Kopenhagener Schule viel Kritik von Vertretern der Critical Security Studies (vgl. Kapitel 2.1.1).

Der zweite Unterschied zur Kopenhagener Schule ist der normative Ansatz der Waliser Schule. ${ }^{58}$ Wie oben bereits erwähnt, haben die Critical Security Studies in der Tradition der Frankfurter Schule ${ }^{59}$ und des Postmarxismus ein emanzipatives Ziel. ${ }^{60}$ Die Waliser Schule versteht Sicherheit als Befreiung von Unterdrückung: "Emancipation, theoretically, is security. ${ }^{61}$ Anders gesagt, nur wenn emanzipatorische Politik die strukturellen Unterdrückungen

56 Vgl. Klüfers, Philipp (2014): Internationale Sicherheit: Neuere Ansätze 3. In: Enskat, S./Masala, C. (2014): Internationale Sicherheit, Wiesbaden: Springer Fachmedien. S. $177 f$.

57 Vgl. Eriksson, Johan (1999): Observers or advocates? On the political role of security analysts. In: Cooperation and Conflict, 34 (3). S. 318.

58 So fungieren die Theoretiker der Waliser Schule nicht nur als Analysten der politischen Ordnungen, sondern fordern in ihren Werken mitunter bestimmte politische Maßnahmen ein. So argumentiert beispielsweise Ken Booth in seinem Buch »Terror in our time«, in der politischen Bekämpfung des internationalen Terrorismus den Islam nicht als das Problem und Demokratie nicht als die Lösung zu betrachten. Vgl. Booth, Ken/Dunne, Tim (2012): Terror in our time. New York: Routledge. S. 11.

59 Während sich Ken Booth stark auf Horkheimer, Adorno und Habermas beruft, orientiert sich Richard Wyn Jones vor allem an Antonio Gramsci. Vgl. Welch, David A. (2000): Review: Security, Strategy and Critical Theory by Richard Wyn Jones. In: The American Political Science Review, Vol. 94, No. 2. S. 523.

60 Für den Emanzipationsbegriff der Waliser Schule siehe: Booth, Ken (2007): Theory of world security, Cambridge [u.a.]: Cambridge Univ. Press. S. 112.

61 C.A.S.E. Collective (2006): Critical Approaches to Security in Europe: A Networked Manifesto. In: Security Dialogue vol 37, no. 4, December 2006. S. 456. 
unserer Zeit beseitigt, kann in der Weltgemeinschaft Sicherheit verwirklicht werden. $^{62}$

Hier liegt der Hauptunterschied zur Kopenhagener Schule. Während Versicherheitlichung und somit auch Sicherheit für die Kopenhagener Wissenschaftler kein positives Gut ist, das es zu erstreben gilt, erlangt Sicherheit durch die Verknüpfung mit Emanzipation aus der Waliser Perspektive einen positiven Wert an sich.

»Uncovering the realities of security (or rather insecurity) entails locating human rights abuses, the opression of minorities, the powerlessness of the poor and violence against women. $\ll^{63}$

Dieser Unterschied zeigt auf, wieso die Waliser Schule für eine Untersuchung von Bürgerwehren ungeeignet ist. Eine kritische Perspektive auf Sicherheit und die Prozesse, die sie hervorbringen, ist unabdingbar, will man nicht Gefahr laufen, Bürgerwehren zu legitimieren - wie McSweeney es der Kopenhagener Schule (fälschlicherweise) vorwarf.

Auch andersherum geht die von Ken Booth aufgestellte Gleichung Sicherheit = Emanzipation in einigen Fällen nicht auf. So können soziale Kämpfe subalterner Gruppen eine emanzipatorische Wirkung entfalten, während sie gleichzeitig zu mehr Unsicherheit für diese Gruppen führen. ${ }^{64}$

\subsubsection{The Authority to Speak Security: Der institutionalistische Ansatz der Pariser Schule}

Die dritte Schule der New European Security Theory entstand in Frankreich und wird vor allem von Didier Bigo repräsentiert. Bigo analysiert den Prozess der Professionalisierung in westlichen Gesellschaften. In diesem Prozess bekommen nach Bigo Akteure der Bürokratie eine zentrale Rolle, wenn es um die Entstehung von Sicherheit geht. Gesellschaftliche Bedrohungen resultieren aus Sicht der Pariser Schule aus bürokratischen Routinen der Kategori-

62 Booth, Ken (2007): Theory of world security. Cambridge [u.a.]: Cambridge Univ. Press. S. 114.

63 C.A.S.E. Collective (2006): Critical Approaches to Security in Europe: A Networked Manifesto. In: Security Dialogue vol 37, no. 4, December 2006. S. 456.

64 Vgl.ebd. S. 456. 
sierung, Einordnung und Definition von Begebenheiten. ${ }^{65} \mathrm{Im}$ Gegensatz zur Kopenhagener Schule ist diese Theorie also wesentlich institutionalistisch.

Für die Pariser Wissenschaftler ist »Sicherheit (...) nicht das Gegenteil von Unsicherheit. ${ }^{66}$ Wie Sicherheit gesellschaftlich und politisch definiert wird, bestimmt, was als unsicher empfunden wird. Dabei konkurrieren verschiedene Sicherheitsexpert"innen innerhalb bürokratischer Strukturen (security agencies) um die Möglichkeit, etwas als Sicherheitsproblem zu benennen (labelling). Die security agencies haben unterschiedlich große symbolic power, die ihnen ermöglicht, ihre Sicht der Sicherheit im Diskurs zu platzieren. Bei der Konzeption der symbolic power beruft sich Bigo auf Pierre Bourdieu. Datenbanken, Statistiken und informationstechnische Ressourcen der einzelnen Akteure dienen als Ressourcen der symbolic power und entscheiden, in zahlreichen informellen Kämpfen zwischen einzelnen Akteuren innerhalb der konkurrierenden bürokratischen Strukturen, wessen Bedrohungsklassifikation die Sicherheitsdebatte letztlich dominiert. ${ }^{67}$

In ihrer Konzeption beruft sich die Pariser Schule dezidiert auf die Kopenhagener Schule und grenzt sich von ihr ab. So setzt Bigo der Unterscheidung Wævers zwischen state und societal security eine Unterscheidung zwischen externer und interner Sicherheit entgegen.

»External security agencies (the army, the secret service) are looking inside the borders in search of an enemy from outside. (...) Internal security agencies (national police forces, police with military status, border guards, customs) are looking to find their internal enemies beyond the borders and speak of networks of crime. ${ }^{68}$

65 Vgl. Klüfers, Philipp (2014): Internationale Sicherheit: Neuere Ansätze 3. In: Enskat, S./Masala, C. (2014): Internationale Sicherheit, Wiesbaden: Springer Fachmedien. S. 202.

66 Vgl. C.A.S.E. Collective (2006): Critical Approaches to Security in Europe: A Networked Manifesto. In: Security Dialogue vol 37, no. 4, December 2006. S. 457.

67 Vgl. Klüfers, Philipp (2014): Internationale Sicherheit: Neuere Ansätze 3. In: Enskat, S./Masala, C. (2014): Internationale Sicherheit. Wiesbaden: Springer Fachmedien. S. 202.

68 Bigo, Didier (2000): When two become one. Internal and external securitizations in Europe. In: International Relations Theory and the Politics of European Integration, edited by Kelstrup, Morten/Williams, Michael C. London/New York: Routledge. S. 171204. Zitiert nach: www.didierbigo.com/students/readings/When \%20Two \%20Become \%20One.pdf S. 320. 
Die Unterscheidung zwischen interner und externer Sicherheit verschwimmt jedoch mitunter in Bigos Ansatz. ${ }^{69}$

Die Pariser Schule steht - wohl auch wegen sprachlicher Barrieren - in der Theoriedebatte der New European Security Studies etwas abseits. Dennoch konnte Didier Bigo eine beträchtliche Lücke in der Theorie der Versicherheitlichung nach Buzan und Wæver schließen. Die Kopenhagener Theorie der Versicherheitlichung hat Probleme, zu erklären, wer auf einer gesellschaftlichen Ebene in einer Position ist, für die Gesellschaft $\mathrm{zu}$ sprechen. Denn sobald die politische Ebene verlassen wird, kann die Frage: »Who is in a powerful position to speak security? « nicht mehr einfach mit einem Verweis auf Politikerinnen und Politiker beantwortet werden. ${ }^{70}$ Bigo schließt diese Lücke mit einem Institutionalismus, der zwar eine schlüssige Antwort auf die genannte Frage liefert, jedoch zivilgesellschaftliche Phänomene wie Bürgerwehren nicht zu erfassen vermag. Der Fokus auf in bürokratischen Strukturen handelnde Akteure lässt die Pariser Schule also für eine Analyse von Bürgerwehren weniger geeignet erscheinen als die Kopenhagener Schule, weil Bürgerwehren zunächst autonome subkulturelle Erscheinungen mit geringem Organisations- und Institutionalisierungsgrad darstellen.

Allerdings findet sich bei der Pariser Schule eine stärkere soziologische Einbettung des Sicherheitsbegriffs als bei der Kopenhagener Schule. Nicht der einzelne Sprechakt steht im Vordergrund, sondern eine komplexere soziale Praxis. ${ }^{71}$ So kritisiert Bigo an Buzan und Wæver, dass sich der Prozess der Versicherheitlichung nur auf den Sprechakt konzentriert:

»[Securitization theory] omits all that is of semiotic interest, such as gestures, manoeuvres, the rituals of demonstration of force which are of cause fundamental in the economy of securitization. ${ }^{72}$

\section{Ebd., S. 342.}

70 Vgl. Hinz, Anne (2007): Security and the Costs and Benefits of Manipulating Analytical Boundaries. Constructivist Debates Within European Critical Security Studies. In: Sicherheit und Frieden (S+F)/Security and Peace 25 (4). Nomos Verlagsgesellschaft $\mathrm{mbH}$. S. 206.

71 Vgl. Büger, C./Stritzel, H. (2005): New European Security Theory. Zur Emergenz eines neuen europäischen Forschungsprogramms. In: Zeitschrift für Internationale Beziehungen. Nomos Verlagsgesellschaft. S. 440.

72 Bigo, Didier (2000): When two become one. Internal and external securitizations in Europe. In: International Relations Theory and the Politics of European Integration, edited by Kelstrup, Morten/Williams, Michael C. London/New York: Routledge. S. 194. 
Die Kopenhagener Schule reagierte jedoch auf die Kritik, dass ihr Fokus auf den reinen Sprechakt zu eng gefasst sei, und führte das Konzept der "performative action « ein (siehe auch Kapitel 2.2). ${ }^{73}$ Das ist für diese Untersuchung insofern wichtig, als bei Bürgerwehren zwar die Rhetorik zentral ist, mit der ihr Bestehen gerechtfertigt wird, jedoch auch nonverbale Handlungen wie ein martialisches Auftreten auf den Patrouillen auf die Bedrohungswahrnehmung durch die audience wirken. Die Herangehensweise der Kopenhagener Schule ist insofern sehr gut für die Untersuchung von Bürgerwehren geeignet.

\subsection{Bürgerwehren als Akteure im Versicherheitlichungsprozess}

Wie in Kapitel 2.1 gezeigt, ist die Theorie der Versicherheitlichung, wie sie die Kopenhagener Schule formuliert, für eine Untersuchung von Bürgerwehren besser geeignet als die Waliser oder die Pariser Schule. Zum einen können die securitizing actors neben Staaten auch gesellschaftliche Akteure und "ganz normale« Individuen sein und nicht - wie dies bei der Pariser Schule der Fall ist nur Personen mit einer Funktion innerhalb gesellschaftlicher Institutionen.

Auch ist der Sicherheitsbegriff nicht - wie dies bei der Waliser Schule der Fall ist - auf das Individuum gerichtet, sondern auf die Gemeinschaft. ${ }^{74}$ Bürgerwehren wollen nicht sich selber schützen, sondern die Gemeinschaft. Und schließlich ist Sicherheit in der Theorie der Kopenhagener Schule, anders als für die Waliser Schule, kein positives Gut, das es zu erstreben gilt, und ermöglicht somit, die Gründung von Bürgerwehren als Versicherheitlichungsprozesse kritisch zu betrachten.

Im Folgenden wird daher die Theorie der Versicherheitlichung im Hinblick auf eine Anwendung auf Bürgerwehren in Deutschland diskutiert. Inwiefern lässt dich das Phänomen der Bürgerwehren mithilfe der Theorie erklären? Oder anders formuliert, lassen sich die Gründungen von Bürgerwehren als Versicherheitlichungsprozesse beschreiben? Zunächst gilt es zu erörtern, ob die Mitglieder einer Bürgerwehr als Sprecher*innen fungieren kön-

73 Vgl. Stritzel, Holger (2007): Towards a Theory of Securitization: Copenhagen and Beyond. In: European Journal of International Relations, Vol. 13(3). S. 357-383.

74 Vgl. Klüfers, Philipp (2014): Internationale Sicherheit: Neuere Ansätze 3. In: Enskat, S./Masala, C. (2014): Internationale Sicherheit, Wiesbaden: Springer Fachmedien. S. 179. 
nen, die mit der Gründung ihrer Bürgerwehr einen Sprechakt der Versicherheitlichung ausüben können. Auf den Punkt gebracht: Sind die Mitglieder einer Bürgerwehr securitizing actors?

In den meisten Arbeiten, in denen das Konzept der Versicherheitlichung angewendet wird, ist der Akteur der Staat oder staatliche Eliten. In Sicherheitsfragen gelten staatliche Akteure als besonders kompetent, weil Sicherheit, spätestens seit der Neuzeit, als staatliche Aufgabe aufgefasst wird. Es können allerdings auch gesellschaftliche Akteure Sicherheitsprobleme benennen, diese verfügen jedoch meist über einen gewissen Organisationsgrad und eine relevante gesellschaftliche Position.

Im Falle von Bürgerwehren ist das nicht immer der Fall. Bürgerwehren sind subkulturelle Erscheinungen und werden meist nur nach längerem Bestehen zu institutionalisierten Akteuren - zum Beispiel, wenn der Staat sogenannte "Sicherheitspartnerschaften« mit ihnen schließt und sie nach einer Schulung mit geringfügigen Polizeiaufgaben betraut. Wæver sieht das Problem ebenfalls und fragt: »How does society speak? $\ll^{75}$ Weil die Gesellschaft im Gegensatz zum Staat keine formellen Institutionen der Repräsentation habe, könne prinzipiell jede und jeder im Namen der Gesellschaft sprechen. Woran macht man also fest, wer als securitizing actor in der Gesellschaft fungieren kann?

Gesellschaften sind keine homogenen Akteure. Es geht nicht darum, ob Bürgerwehren für die lokale Bevölkerung sprechen. Vielmehr muss Sprecher"in hier im weiteren Sinne verstanden werden. Mehrere Sprecher"innen können ineinandergreifen, und auch Bilder, beispielsweise in der medialen Berichterstattung, können zu Versicherheitlichungen beitragen. Die Kopenhagener Schule erweiterte ihr Konzept jedoch entsprechend und legte dem Versicherheitlichungsprozess eine breitere kommunikative Handlung (performative action) zugrunde. ${ }^{76}$

Für die Untersuchung von Bürgerwehren ist die Erweiterung des Konzepts des Sprechaktes hin zur performative action zentral. Bürgerwehren agieren mitunter bereits durch ihr physisches Auftreten. Bereits der Akt, gemeinsam durch die Straßen zu laufen, ist Teil der Versicherheitlichung. Dennoch

75 Vgl. Wæver, Ole (1995): Securitization and Desecuritization. In: Lipschutz, Ronnie D. (Hg.): On Security. New York: Columbia University Press. S. 69.

76 Vgl. Stritzel, Holger (2007): Towards a Theory of Securitization: Copenhagen and Beyond. In: European Journal of International Relations, Vol. 13(3). S. 357-383. 
bleibt der Sprechakt zentral, denn ohne die Aussage, »wir sind eine Bürgerwehr« oder »wir stellen die Sicherheit wieder her«, wäre die Patrouille nichts weiter als ein Spaziergang einiger Bürger*innen.

Für die Analyse von Bürgerwehren ist also der Sprechakt, verstanden als eine performative action, relevant. Die Sprache bleibt der zentrale Fokus, kann aber durch Handlungen ergänzt werden. Dabei geht es mehr um die Logik des Sprechaktes als um den/die Sprecher*in und ihre Handlungen selbst:

»How to identify the securitizing actors is in the last instance less a question of who performs the speech than of what logic shapes the action. « ${ }^{77}$

Dennoch muss der/die Sprecher*in nach Buzan/Wæver eine position of authority innehaben. Es bleibt das Problem, dass die Mitglieder einer Bürgerwehr nicht unbedingt herausragende soziale Positionen innerhalb der Gemeinschaft innehaben. Aber Menschen, die eine Bürgerwehr gründen wollen, haben ein Interesse daran, als kompetente Akteure im von ihnen artikulierten Sicherheitsproblem zu erscheinen, denn sie wollen sich schließlich auch selber als Lösung positionieren. Ihre Patrouillen sollen die Sicherheit wiederherstellen.

Entweder sind sie schon relevante Persönlichkeiten und erleichtern durch ihre Stellung die Gründung einer Bürgerwehr ${ }^{78}$ oder sie werden es durch den Aufruf zur Gründung einer Bürgerwehr. Letzterer Fall ist in der Theorie der Versicherheitlichung nicht vorgesehen. Jedoch wäre er leicht durch eine konsequente und die Theorie nicht infrage stellende Erweiterung der möglichen Sprecher*innen integrierbar. Sollte sich während der Untersuchung herausstellen, dass Sprecher*innen erfolgreich Versicherheitlichungen herbeiführen können, die, bevor sie ihre Stimme erhoben, keine relevante soziale Position in der Gesellschaft innehatten, so wird die Theorie der Versicherheitlichung in dieser Hinsicht erweitert werden müssen.

Ob Bürgerwehren securitizing actors sein können, hängt auch davon ab, ob sie für die Gesellschaft sprechen können. Mit Gesellschaft ist hier die örtliche Bevölkerung gemeint, an die sich der Sprechakt richtet, die Gemeinschaft,

77 Buzan/Wæver/de Wilde (1998): Security. A New Framework for Analysis. London/Colorado: Rienner. S. 41.

78 So hat beispielsweise in Lawitz die Bürgermeisterin des Ortes die Bürgerwehr mitgegründet: Thurm, Frida (2016): Unter Kontrolle. In: Die Zeit. Online unter: https:/ /www.zeit.de/feature/buergerwehr-brandenburg-diebe-polen-fluechtlinge(Zugriff am 2.1.2021). 
innerhalb derer ein Thema durch die Gründung einer Bürgerwehr versicherheitlicht wird. Das Sprechen für die örtliche Bevölkerung wird dabei nicht im Sinne eines Konsenses verstanden; vielmehr ist es eine Mischung aus offener Zustimmung und stillschweigender Unterstützung. Die Unterstützung für die Bürgerwehr erfahren ihre Mitglieder über soziale Medien, informelle Gespräche zwischen Nachbar*innen und natürlich durch einen Zulauf zur Bürgerwehr.

Der existential threat, den die Mitglieder einer Bürgerwehr artikulieren, ist je nach Bürgerwehr unterschiedlich. Oft sind es häufige Einbrüche in der Nachbarschaft, aber auch abweichende Verhaltensweisen (Exhibitionisten, Wildpinkler) oder Migranten und Geflüchtete. Insbesondere nach den Übergriffen in der Silvesternacht 2015/16 am Kölner Hauptbahnhof (\#koelnhbf) gründeten sich zahlreiche Bürgerwehren mit dem Vorhaben, »unsere Frauen« vor »nordafrikanischen/muslimischen Männern« zu schützen. Diese Bürgerwehren lassen sich jedoch alle dem rechten Spektrum zuordnen. Ihre Gründung erfolgte ebenso sehr als politische Aktion, um ihren Rassismen Ausdruck zu verleihen.

Die Akteure, also die Mitglieder der Bürgerwehr, können dabei an eine politization anknüpfen. Ein Thema muss also schon politisiert sein, um versicherheitlicht werden zu können. Im Falle von \#koelnhbf ist diese politization eindeutig in der öffentlichen Debatte festzustellen. Aber auch in anderen Fällen lässt sich diese politization beobachten. Wenn beispielsweise die Lokalpresse viel über Einbrüche in der Region berichtete und die fehlende Polizeipräsenz bemängelte, fällt es einer Bürgerwehr leichter, die Einbrüche zu einem Sicherheitsthema zu machen. Ebenso können die örtliche Stimmung, Nachbarschaftsgespräche und Facebook-Diskussionen eine solche politization bewirken.

Die Bedrohung, die vom Akteur wahrgenommen und benannt wird, bezieht sich auf ein referent object, welches durch die Bedrohung in Gefahr ist. Im Falle der Einbrüche wäre das Referenzobjekt das Eigentum und die Unversehrtheit der Wohnung. Bei den Bürgerwehren, die sich als Reaktion auf \#koelnhbf gründeten, ist das zugrunde liegende referent object »die deutschen Frauen« sowie eine von den Mitgliedern der Bürgerwehr konstruierte »deutsche Identität«.

Hier zeigt sich, wie relevant die von McSweeney angestoßene Debatte für die Anwendung der Theorie der Versicherheitlichung auf das Phänomen Bürgerwehr ist. McSweeney kritisierte, das Identitätskonzept von Buzan und Wæver sei zu essentialistisch und laufe somit Gefahr, nationalistische Bewe- 
gungen zu legitimieren (vgl. Kapitel 2.1.3). Ein starres Konzept von Identität eignet sich jedoch genau für die Untersuchung von Bürgerwehren besonders gut, da sich Bürgerwehren selber, wenn sie ihr Bestehen mit einer lokalen/nationalen/kulturellen Identität, die es zu sichern gelte, rechtfertigen, eines Konzepts bedienen, das Identität als Entität begreift. Hier finden sich keine Relativierungen oder Verweise auf die Prozesshaftigkeit von Identität. Diese Sicht von Identität darf jedoch nicht positivistisch als wahre oder einzige Identität verstanden werden. Vielmehr dient sie dem Verständnis der Argumentation des securitizing actors in seinem Versuch, eine Versicherheitlichung herbeizuführen.

Ginge es um die Identität der Mitglieder der Bürgerwehr selber, sähe die Sache anders aus. Hier müsste ein Konzept komplexer und prozesshafter ausgestaltet sein. Diese Untersuchung ist jedoch nicht Teil der Studie. Hier wird untersucht, ob Bürgerwehren die Normen der Gemeinschaft bedroht sehen, und ob sie eine »in-group« vor einem bedrohlichen Außen schützen wollen. Denn die von den Bürgerwehren artikulierten Sicherheitsbedrohungen kommen meist nicht aus dem Ort: Einbrecher*innen, »osteuropäische Banden«, »Flüchtlinge«.

Das relevante Publikum (audience), an das der Sprechakt adressiert ist, wäre im Fall der Bürgerwehren die örtliche Bevölkerung. Jedoch verschwimmen auch hier die Grenzen, weil sich auch das Publikum jederzeit der Bürgerwehr anschließen kann. Zudem ließe sich an dieser Stelle einwenden, dass Bürgerwehren gar kein Publikum brauchen. Sie sind eine subkulturelle Erscheinung, die sich in einer kleinen in-group bewegt und die extraordinary measures einfach in Angriff nimmt - auch ohne eine Anerkennung der Bedrohung durch ein relevantes Publikum. Mit anderen Worten: Eine Bürgerwehr geht auf $\mathrm{Pa}$ trouille und interessiert sich nicht dafür, ob sie dazu befugt wurde, ob die Gesellschaft die von ihr benannten Bedrohungen akzeptiert oder nicht.

Doch sind Bürgerwehren langfristig von der Anerkennung des lokalen Umfelds abhängig. In ländlichen Gegenden ist eine Unterstützung der Nachbar*innen und der lokalen Öffentlichkeit wichtig. In urbanen Räumen bekommen Bürgerwehren Zuspruch und Zulauf vor allem über soziale Netzwerke wie Facebook. Neue Interessierte, die auf Patrouille gehen wollen, können hier unkompliziert Kontakt zur Bürgerwehr aufnehmen. Bürgerwehren wenden sich mit einer existentiellen Bedrohung also an die lokale Bevölkerung (audience), um ihr Bestehen zu legitimieren.

Die Legitimationsmuster der Mitglieder werde ich mittels Interviews mit den Bürgerwehren erfassen. Versicherheitlichungsprozesse nachzuweisen, 
erfordert die Suche nach einer spezifischen rhetorischen Struktur innerhalb von Diskursen, durch die etwas als existentielle Bedrohung platziert wird. ${ }^{79}$ Die Reaktion der audience auf den Sprechakt analysiere ich anhand von Reaktionen des Umfelds, der lokalen Bevölkerung, der örtlichen Politik sowie anhand von Resonanz (likes/shares) in den sozialen Medien.

Die Sicherheitsmaßnahmen (extraordinary measures) sind, neben der Gründung der Bürgerwehr selbst, vor allem Patrouillen, aber auch potentielle Gewaltanwendungen und im Allgemeinen alle Handlungen der Bürgerwehr, die ergriffen werden mit der Begründung, Sicherheit herzustellen. Der Sprechakt (und somit die Versicherheitlichung des von der Bürgerwehr als Grund für ihre Gründung angeführten Themas) ist erfolgreich, wenn die Bürgerwehr sich innerhalb der örtlichen Bevölkerung auf Zeit als Akteur etablieren kann.

\subsection{Die Vergeschlechtlichung des Versicherheitsprozesses}

Die New European Security Theory zeichnet sich durch eine Ausklammerung der Dynamik aus, die Männlichkeiten in Versicherheitlichungsprozessen entwickeln. Zwar existieren Studien, die eine Versicherheitlichung von Phänomenen untersuchen, in denen die (soziale) Kategorie Geschlecht eine Rolle spielt, ${ }^{80}$ aber der strukturelle Einfluss auf den Versicherheitlichungsprozess selbst ist bisher nicht betrachtet worden.

Dabei stellen Bürgerwehren einen idealen Untersuchungsgegenstand dar, um die Vergeschlechtlichung von Versicherheitlichungsprozessen aufzuzeigen. Erstens, weil die Akteure des Versicherheitlichungsprozesses Individuen sind; zweitens, weil der Akt des Beschützens, der jedem Versicherheitlichungsprozess innewohnt, nicht von Institutionen übernommen wird, sondern direkt an die Körper der Akteure des Versicherheitlichungsprozesses gebunden ist. Die Mitglieder der Bürgerwehren erlangen mit dem Akt der Benennung einer Bedrohungslage die Möglichkeit, sich zum Beschützer zu

79 Vgl. Bonacker, T./Bernhardt, J. (2014): Von der security community zur securitized community. In: Siedschlag, A. (2014): Methoden der sicherheitspolitischen Analyse. Eine Einführung. Wiesbaden: VS Verlag. S. 247.

80 So untersucht Carpenter beispielsweise die Versicherheitlichung von »gender-based violence«: Vgl. Carpenter, R. (2006): Recognizing Cender-Based Violence Against Civilian Men and Boys in Conflict Situations. Security Dialogue, 37(1). S. 83-103. 
erklären, der auf den Straßen für Sicherheit sorgt. Diese vergeschlechtlichte Motivation für Versicherheitlichungsprozesse lässt sich anhand von ethnographischen Einzelfallstudien über Bürgerwehren besonders plastisch zeigen.

Zudem ist in einem der drei untersuchten Fälle das referent object selbst vergeschlechtlicht. Die Bürgerwehr in Weißensee möchte Frauen beschützen. Hier ist die Motivation für die Gründung der Bürgerwehr, also für den Versicherheitlichungsprozess, direkt von Vorstellungen über Weiblichkeiten und Männlichkeiten geprägt. Zwar ist eine theoretisch fundierte Analyse der Geschlechterordnung, die innerhalb von Bürgerwehren performt wird, im Rahmen dieser Untersuchung nicht möglich, dennoch lassen sich aus dem Verhalten der Mitglieder der untersuchten Bürgerwehren sowie aus ihren Legitimationsdiskursen Erkenntnisse über den Einfluss generieren, den Männlichkeiten auf den Versicherheitlichungsprozess haben. Daher werden im folgenden Unterkapitel zunächst die Konstruktionen verschiedener Männlichkeiten nach Raewyn Connell beschrieben. Anschließend wird das Konzept im Hinblick auf Dynamiken im Versicherheitlichungsprozess erörtert, um eine Betrachtung der untersuchten Bürgerwehren im Hinblick auf den Einfluss von Männlichkeiten auf Versicherheitlichungsprozesse zu ermöglichen.

\subsubsection{Hegemoniale und marginalisierte Männlichkeiten}

Der Betrachtung von Männlichkeiten liegt hier die Annahme zugrunde, dass Geschlechtsmerkmale nicht nur auf biologische Einflüsse zurückgehen, sondern auch auf soziale. Geschlecht als soziale Kategorie ist abhängig vom soziokulturellen Kontext, in dem es erlernt wurde. Es gibt daher nicht nur eine Männlichkeit. Und bei der Formierung verschiedener Männlichkeiten stehen Männer nicht nur im Verhältnis zu Frauen, sondern auch zu anderen Männern. Daraus entsteht nach Raewyn Connell eine hierarchische Binnenschichtung innerhalb der Gruppe der Männer. Sie unterscheidet hier zwischen vier Männlichkeiten: untergeordneter, marginalisierter, komplizenhafter und hegemonialer Männlichkeit. ${ }^{81}$

81 Zur grundlegenden Darlegung der Theorie vgl. Connell, Raewyn (2015): Der gemachte Mann, Geschlecht und Gesellschaft. Konstruktion und Krise von Männlichkeiten, 4. erw. Aufl. Wiesbaden: Springer Fachmedien. Connell hat seine Theorie jedoch beständig weiterentwickelt und auf Kritik reagiert. Insbesondere verweisen die neueren Analysen darauf, dass es nicht nur eine hegemoniale Männlichkeit gibt, und dass Männlichkeitsforschung im Sinne einer postkolonialen Weiterentwicklung eine 
Hegemoniale Männlichkeit hat Zugang zu den Privilegien des Patriarchats. Diese Gruppe von Männern ist sehr divers und füllt ihre Rolle als dominante soziale Gruppe (sowohl gegenüber Frauen als auch gegenüber anderen Männern) auf unterschiedliche Weise aus. Das verbindende Element ist jedoch, dass sie, um ihre Machtansprüche durchzusetzen, auf Hegemonie ${ }^{82}$ zurückgreifen kann.

»Most accounts of hegemonic masculinity do include such >positive actions as bringing home a wage, sustaining a sexual relationship, and being a father. Indeed it is difficult to see how the concept of hegemony would be relevant if the only characteristics of the dominant group were violence, aggression, and self-centeredness. Such characteristics may mean domination but hardly would constitute hegemony - an idea that embeds certain notions of consent and participation by the subaltern groups. ${ }^{83}$

Weil Macht jedoch sehr konzentriert ist, können nur wenige Männer diese Form von Männlichkeit leben. Eine größere Gruppe macht die komplizenhafte Männlichkeit aus. Sie entsprechen nicht in allen Bereichen der Norm von Männlichkeit als Souveränität, Überlegenheit und Autorität besitzend, dennoch profitieren sie von der Vormachtstellung des Mannes. Sie erhalten eine "patriarchale Dividende«, einen Vorteil, der allen Männern (und auch einigen Frauen) aus der Unterdrückung von Frauen im Patriarchat erwächst. ${ }^{84}$

globale Perspektive auf Männlichkeiten einnehmen muss: Vgl. hierzu: Connell, Raewyn/Wood, J. (2005): Globalization and business masculinities. In: Men and Masculinities 7 (4). S. 347-364. Sowie: Messerschmidt/Martin/Messner/Connell (Hg.) (2018): Cender Reckonings: New Social Theory and Research. New York: NYU Press. Sowie: Connell, Raewyn/Messerschmidt, James (2005): Hegemonic Masculinity: Rethinking the Concept. In: Cender and Society, Vol. 19, No. 6. S. 829-859. Sowie: Connell, Raewyn (2009): Der Sprung über die Kontinente hinweg. Überlegungen zur Entwicklung von Erkenntnismethoden und Ansätzen in der Männlichkeitsforschung. In: Aulenbacher, Brigitte/Riegraf, Birgit (Hg.): Erkenntnis und Methode. Wiesbaden: VS Verlag. S. 81100.

82 Zur Diskussion des Hegomoniekonzepts hinter Connells Unterscheidung siehe: May, Michael (2010): Hegemoniale Männlichkeit. In: Böllert, Karin/Oelkers, Nina (2010) (Hg.): Frauenpolitik in Familienhand? Wiesbaden: VS Verlag. S. 129-156.

83 Vgl. Connell, Raewyn/Messerschmidt, James (2005): Hegemonic Masculinity. Rethinking the Concept. In: Cender and Society, Vol. 19, No. 6. S. 840f.

84 Vgl. Connell, Raewyn (2015): Der gemachte Mann, Geschlecht und Gesellschaft. Konstruktion und Krise von Männlichkeiten, 4. erw. Aufl. Wiesbaden: Springer Fachmedien. S. 133. 
Männer, die benachteiligt sind - beispielsweise Aufgrund anderer Diskriminierungsmechanismen wie Rassismus oder Klassismus -, performen marginalisierte Männlichkeiten. Sie werden von anderen Männern unterdrückt und erleben daher eine Spannung zwischen dem Anspruch, ein Männlichkeitsideal erfüllen zu sollen, das Souveränität, Überlegenheit und Autorität beinhaltet, dabei aber keinen Raum dafür lässt, Opfer zu sein und Schwäche zu zeigen, und persönlich erfahrener Unterdrückung. Marginalisierte Männlichkeit führt $\mathrm{zu}$ fragilen und spannungsgeladenen männlichen Identitätskonstruktionen, die eine Notwendigkeit mit sich bringen, diese fragile Männlichkeit zu stabilisieren. Die Stabilisierungsprozesse können dabei sehr unterschiedlich sein. Ein Aspekt davon kann Gewalt gegen Frauen und untergeordnete Männer sein. ${ }^{85}$ Das soll aber nicht heißen, dass Gewalt nur durch diese Form von Männlichkeit ausgeübt wird.

Die vierte Kategorie bildet die untergeordnete Männlichkeit. Sie wird von Männern performt, die bezüglich der hierarchischen Binnenschichtung von Männern auf der untersten Stufe stehen. Raewyn Connell beschreibt untergeordnete Männlichkeit als diejenige Männlichkeit, die das Patriarchat untergräbt, also Männer, die weiblich konnotiertes Verhalten performen und homosexuelle Männer. Diese Männlichkeit wird von allen drei anderen Männlichkeiten unterdrückt, um die Vormachtstellung des Mannes aufrecht zu erhalten. ${ }^{86}$

Wichtig bei der Unterscheidung der verschiedenen Männlichkeiten ist, dass die fragilen und spannungsreichen Prozesse der Herstellung von Männlichkeiten keinesfalls deterministisch sind. Hegemoniale oder marginalisierte Männlichkeiten stellen keine festen Charaktertypen dar, sondern müssen im Sinne von Handlungsmustern verstanden werden. Für alle Gruppen gilt: Die Frage, ob und für wen die eigene Männlichkeitsidentität fragil und spannungsreich ist, ist empirisch offen. ${ }^{87}$

85 Vgl. ebd. S. 138.

86 Vgl. ebd., S. 199.

87 Bereswill, Mechthild (2009): Marginalisierte Männlichkeit als gesellschaftliche und biographische Konfliktdynamik. Empirische Einsichten und methodologische. In: Aulenbacher, Brigitte/Riegraf, Birgit (Hg.): Erkenntnis und Methode. Wiesbaden: VS Verlag. S. 149. 


\subsubsection{Fragile Männlichkeiten als Motor für Versicherheitlichungsprozesse}

Im Anschluss an Connells Konzept entstanden zahlreiche empirische Studien zu marginalisierter Männlichkeit; ${ }^{88}$ Bürgerwehren in Deutschland und die Dynamiken von Versicherheitlichungsprozessen generell sind jedoch bisher nicht im Hinblick auf Männlichkeiten untersucht worden.

Mithilfe von Connells Unterscheidung verschiedener Männlichkeiten wird der Einfluss der sozialen Kategorie Geschlecht auf Bürgerwehren als Versicherheitlichungsprozesse beschreibbar. Um im Rahmen der Einzelfallstudien die Vergeschlechtlichung des Versicherheitlichungsprozesses herausarbeiten zu können, wird im Folgenden die Struktur des Versicherheitlichungsprozesses, wie sie die Kopenhagener Schule beschreibt, im Hinblick darauf diskutiert, wie sich das Konzept marginalisierter Männlichkeit in den Ablauf des Versicherheitlichungsprozesses integrieren lassen könnte. ${ }^{89}$

Der Versicherheitlichungsprozess beginnt mit einer wahrgenommenen Unsicherheit bezüglich einer Bedrohungslage. Wenn diese Unsicherheiten von einer Gruppe wahrgenommen werden, die eine marginalisierte

88 Für den deutschen Kontext vgl. bspw. Bereswill, Mechthild (2007): Undurchsichtige Verhältnisse. Marginalisierung und Ceschlecht im Kontext der Männlichkeitsforschung. In: Klinger, C./Knapp, G.-A./Sauer, B. (Hg.): Achsen der Ungleichheit-Achsen der Differenz. Verhältnisbestimmungen von Klasse, Ceschlecht, Rasse/Ethnizität. Frankfurt a.M./New York: Campus, 84-99. Und: Neuber, Anke (2009): Die Demonstration kein Opfer zu sein: Biographische Fallstudien zu Cewalt und Männlichkeitskonflikten. Interdisziplinäre Beiträge zur kriminologischen Forschung, Band 35. BadenBaden: Nomos. Sowie: Findeisen, Hans Volkmar/Kersten, Joachim (1999): Der Kick und die Ehre. Vom Sinn jugendlicher Cewalt. München: Antje Kunstmann Verlag. Und: Kersten, Joachim (1997): Risiken und Nebenwirkungen: Cewaltorientierung und die Bewerkstelligung von >Männlichkeit< und >Weiblichkeit < bei Jugendlichen der underclass. In: Kriminologisches Journal, Beiheft 6. S. 103-114. Für einen Überblick über den Stand der empirischen Forschung zu marginalisierten Männlichkeiten siehe auch: Bereswill, Mechthild/Neuber, Anke (2010): Marginalisierte Männlichkeit, Prekarisierung und die Ordnung der Ceschlechter. In: Lutz/Herrera Vivar/Supik (2010) (Hg.): Fokus Intersektionalität. Wiesbaden: VS Verlag. S. 88ff.

89 Ebenso kann auch hegemoniale Männlichkeit Auswirkungen auf den Versicherheitlichungsprozess haben - und umgekehrt kann Versicherheitlichung hegemoniale Männlichkeit stabilisieren. Im Rahmen dieser Studie ist eine Untersuchung dieser Wechselbeziehung jedoch nicht möglich. 
Männlichkeit performt, so verändert dies die Bedingungen für den Versicherheitlichungsprozess. Marginalisierte Männlichkeit reagiert auf Ängste und Unsicherheiten anders als andere soziale Gruppen.

Wahrgenommene Unsicherheiten wirken dabei im Zusammenspiel mit der Fragilität männlicher Identitätskonstruktionen. So ist marginalisierte Männlichkeit Diskriminierungen ausgesetzt, die einem Erfüllen von Männlichkeitsidealen wie Souveränität, Überlegenheit und Autorität im Wege stehen. Um spannungsgeladene Männlichkeitsidentitäten zu stabilisieren, muss auf anderen Ebenen umso mehr Übereinstimmung mit Männlichkeitsattributen hergestellt werden. ${ }^{90}$ Ängste werden dabei als Zeichen von Schwäche interpretiert, die nicht mit den Männlichkeitsansprüchen in Einklang zu bringen sind. ${ }^{91}$ Unsicherheitswahrnehmungen verstärken also das Spannungsverhältnis von nicht-erfülltem Männlichkeitsideal und eigener sozialer Position und den damit einhergehenden Gefühlen. Sie lassen ein potentielles Opfer-werden erkennen, das abgewehrt werden muss, um die Männlichkeitsidentität zu stabilisieren. Die Gründung einer Bürgerwehr ermöglicht Handlungsmacht und das Einnehmen einer Position der Stärke. Zum Beschützer zu werden, wendet sich gegen die Position des Ängstlichen, gegen die des Opfers und stabilisiert die Identität einer marginalisierten Männlichkeit, weil sie sich den Männlichkeitsidealen wieder annähert.

Wenn, wie im Fall der Bürgerwehren, die Akteure der Versicherheitlichung Individuen sind, so werden diese Auswirkungen besonders sichtbar. Die Mitglieder der Bürgerwehren werden im Prozess der Versicherheitlichung mit ihren Körpern zu Sicherheitsgaranten. Sie können die Unsicherheitsgefühle in andere Personengruppen auslagern, die zu schützen sie sich zur Aufgabe machen. Die Rolle des starken Beschützers knüpft hier an die Handlungsschemata marginalisierter Männlichkeit an.

Im Rahmen der Einzelfallstudien wird also zu untersuchen sein, welchen Einfluss Männlichkeiten auf die persönlichen Motivationen der Mitglieder

90 Vgl. hierzu insbesondere die Studien von James Messerschmidt. Bspw. Messerschmitt, James (2001): Masculinities, Crime and Prison. In: Sabo/Kupers/London (2001): Prison Masculinities. Philadelphia. S. 67-72.

91 Vgl. Bereswill, Mechthild (2007): Undurchsichtige Verhältnisse. Marginalisierung und Geschlecht im Kontext der Männlichkeitsforschung. In: Klinger, C./Knapp, G.-A./Sauer, B. (Hg.): Achsen der Ungleichheit-Achsen der Differenz. Verhältnisbestimmungen von Klasse, Geschlecht, Rasse/Ethnizität. Frankfurt a.M./New York: Campus. S. 90. 
haben, eine Bürgerwehr zu gründen beziehungsweise sich ihr anzuschließen, und wie die Handlungsschemata marginalisierter Männlichkeit den Versicherheitlichungsprozess beeinflussen. 